\title{
Un análisis de la tecnopolítica aeroespacial argentina
}

Jonatan Sabando, Regina Sarmiento

y Tomás Hough

Facultad de Ciencias Astronómicas y Geofísicas,

Universidad Nacional de La Plata

\section{Título: Un análisis de la tecnopolítica aeroespacial argentina}

Resumen: Desde la década de 1940 a la actualidad, el desarrollo tecnológico nacional en materia aeroespacial ha tenido múltiples avances y retrocesos. Este trabajo intenta exponer las distintas visiones de sus protagonistas en los diferentes momentos históricos, y sus consecuencias en la matriz aeroespacial nacional. Por su trascendencia tanto en el plano comercial como militar, la tecnología aeroespacial se ve atravesada por múltiples intereses que impactan fuertemente en el sector. Para caracterizar los distintos períodos nos centramos en la manera en que se dispone del sistema científico-militar para la utilización o desarrollo de este tipo de tecnología y cuales son sus logros. De forma tal que podamos concluir las ventajas y desventajas de los distintos regímenes. Palabras clave: Política aeroespacial, Tecnopolítica, politica satelital, coheteria en argentina

\section{Analysis of Argentine aerospace technopolitics}

Abstract: Since the decade of 1940 to the present, the national technological development in aerospace has had multiple advances and setbacks. This work tries to expose the different visions of its protagonists in different historical moments and their consequences in the national aerospace matrix. Because of its commercial and militar importance, aerospace technology is crossed by multiple interests that strongly impact the sector. To characterize the different periods we focus on the way in which the military-scientific system is involved in the use or development of this type of technology and what are its achievements. We finally conclude mentioning the advantages and disadvantages of the different regimes.

Keywords: Aerospace Policy, Technopolitics, satellite politics, rocket in Argentina

\section{Uma análise da tecnopolítica aeroespacial argentina}

Resumo: A partir da década de 1940 até o presente, o desenvolvimento tecnológico nacional na indústria aeroespacial teve vários avanços e retrocessos. Este trabalho procura expor as diferentes visões de seus protagonistas nos diferentes momentos históricos e suas conseqüências na matriz aeroespacial nacional. Devido à sua importância comercial e militar, a tecnologia aeroespacial é atravessada por múltiplos interesses que impactam fortemente o setor. Para caracterizar os diferentes períodos, focamos na maneira como o sistema científico-militar está disponível para o uso ou desenvolvimento deste tipo de tecnologia e quais são suas realizações. De tal forma que podemos concluir as vantagens e desvantagens dos diferentes regimes. Palavras-chave: Política Aeroespacial, Tecnopolítica, politica satelital, coheteria en Argentina 


\section{Introducción}

El camino recorrido por nuestro país en materia aeroespacial, es como mínimo, turbulento. De los primeros proyectiles en la década de 1940 hasta el lanzamiento del satélite SAOCOM 1A hace algunos meses, hay un camino de múltiples avances y retrocesos. El sistema científico-tecnológico nacional que impulsó esta tecnología no solo está compuesto por científicos y técnicos, también lo integran militares, dirigentes políticos, economistas, instituciones y empresas, tanto estatales como privadas. Esto se debe a que los desarrollos tecnológicos aeroespaciales tienen una finalidad dual: no sólo son preciados por su alto valor agregado sino también por su gran valor estratégico militar. Esta característica genera un complejo entramado en la toma de decisiones, que abarcan distintas visiones en distintos momentos históricos. Ante tal complejidad haremos énfasis en algunos conceptos analíticos que nos servirán como guía para recorrer de manera simplificada este camino.

Tomaremos el concepto de tecnopolítca de Hecht, para caracterizar "la práctica estratégica de diseñar o utilizar la tecnología para constituir, encarnar o impulsar objetivos políticos". Dice la historiadora: "Estos regímenes tecnopolíticos, basados en instituciones, consisten en vínculos entre grupos de gente, prácticas ingenieriles e industriales, artefactos tecnológicos, programas políticos, e ideologías institucionales, los cuales actúan juntos para gobernar los desarrollos tecnológicos y perseguir tecnopolíticas". Y agrega que el concepto de régimen tecnopolítico "provee una buena aproximación a la estrecha relación entre las instituciones, sus dirigentes, los mitos e ideologías que los guían, los artefactos que producen, y la tecnopolítica que persiguen" (Hecht, 1998).

Dado su carácter dual, De león (2015) aclara que el concepto de tecnopolítica es necesario adaptarlo "para un contexto de país semi-periférico, en el cual las relaciones de poder, manifestadas en forma de presiones formales e informales ejercidas por los países centrales, le da a las relaciones internacionales un lugar crucial en la comprensión de los procesos de desarrollo de este tipo de tecnologías".

\section{Los inicios (1940-1958)}

Los primeros pasos de Argentina en materia aeroespacial se dan a fines de la década del 40, cuando las Fuerzas Armadas desarrollan el primer motor de combustible líquido para propulsar proyectiles. En ese momento sólo los Estados Unidos, la Unión Soviética, Inglaterra, Francia y Alemania habían experimentado con un sistema similar. Este hito es una referencia clara del nivel técnico que se poseía en este momento y del interés del gobierno peronista en el desarrollo aeronáutico y en nuevos métodos de propulsión, como la cohetería.

A partir de la llamada Revolución Libertadora, que derrocó a Perón en 1955, la mayoría de los proyectos aeroespaciales fueron cancelados. Sólo por poner un ejemplo, la fabricación del Pulqui II fue reemplazado por la adquisición de aviones estadounidenses F86 Sabre, remanente de la guerra de Corea.

3. Periodo autonomista (1958-1982)Desde la puesta en órbita del Sputnik I, los militares aeronáuticos argentinos gestaron la idea de poner un satélite en el espacio con medios propios. Esa decisión 
| Ciencia, Tecnología y Política | Año 2 | N² | Enero-Junio 2019 | ISSN 2618-3188 | www.revistas.un|p.edu.ar/CTyP |

marcó el inicio del primer régimen tecnopolítico en el área espacial en la Argentina. Este primer régimen tecnopolítico se caracterizó por la búsqueda de un desarrollo autónomo de tecnología espacial. Lo que supuso el desarrollo propio de una tecnología nacional, utilizando una estrategia incremental, para lograr autonomía tecnológica; lo cual implica "pensar soluciones en términos de soberanía y desarrollo. Se trata de implementar, por ejemplo, tecnologías que no estén atadas a multinacionales en la cadena, sino que las pequeñas empresas que las utilizan puedan moverse con cierta autonomía" (Bilmes, 2012).

En 1960 se crea la Comisión Nacional de Investigaciones Espaciales (CNIE), comandada por el ingeniero Teófilo Tabanera'. A partir de este año, se iniciaron actividades espaciales en forma sistemática.

El primer cohete de investigación argentina, el Alfa Centauro, lanzado al espacio el 2 de febrero de 1961, fue desarrollado por un equipo de ingenieros del Instituto Aerotécnico bajo la dirección de Aldo Zeoli². Al mismo tiempo, el Instituto se reestructuró y convirtió en el Instituto de Investigación Aeronáutica y Espacial (IIAE), dependiente de la Dirección Nacional de Fabricación e Investigación Aeronáutica (DINFIA).

Ese mismo año, se crea el Centro de Experimentación y Lanzamiento de Proyectiles Autopropulsados
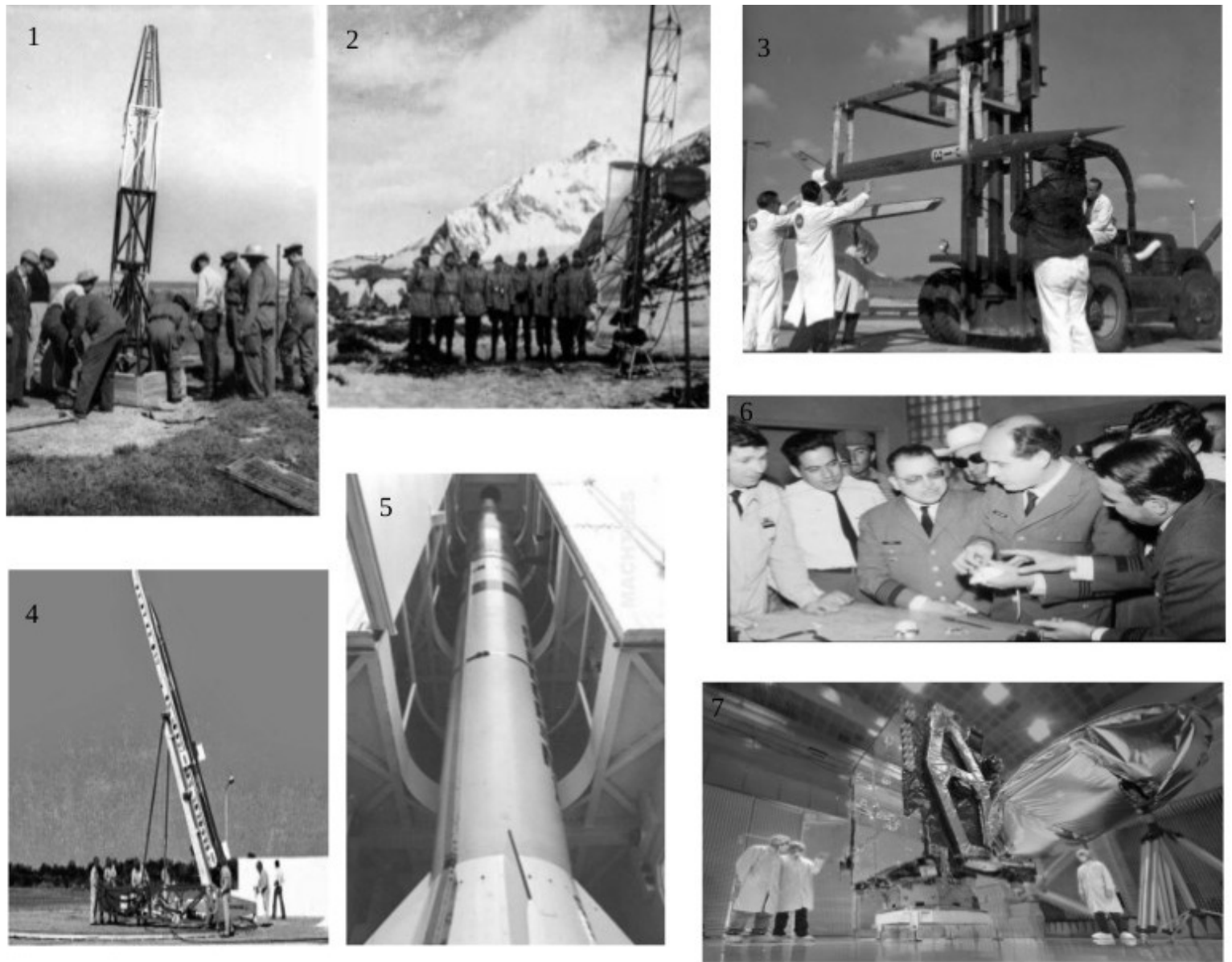

1.Alfa Centauro 2.Operación Matienzo 3.Orión II 4.Tauro 5.Cóndor II 6.Zeoli presenta a Belisario 7.ARSAT II

\footnotetext{
1 Teófilo Tabanera (1909 - 1981) oficial aéreo e ingeniero electromecánico argentino, autor de libros como ¿¿Qué es la astronáutica?'. Fue miembro en la Sociedad Británica Interplanetaria y la Sociedad Americana de Cohetes. Primer presidente de la CNIE e impulsor de la educación a distancia usando tecnología satelital.

${ }^{2}$ Aldo Zeoli (1916 - 2003) comodoro e ingeniero argentino, dirigió el proyecto del primer cohete nacional, promovió la creación de la base CELPA de Chamical y Mar Chiquita, impulsó el proyecto BIO y lanzamientos desde la antártida.
} 
Chamical (CELPA) en La Rioja, donde "tuvieron lugar las primeras experiencias con la utilización de tres plataformas espaciales: cohetes-sonda Beta y Gamma Centauro y balones estratosféricos" (Vera, Guglielminotti, y Moreno; 2015)

Con el golpe de estado al presidente Frondizi, en 1962, la Fuerza Aérea se hizo cargo de la CNIE. A partir de este año, el IIAE diseñó y fabricó cohetes aceleradamente. Se desarrollaron varias series denominadas Orión, Canopus, Rigel, Castor y Tauro. Algunos entraron en servicio de las Fuerzas Armadas posteriormente.

Hitos importantes durante los primeros años de la CNIE fueron: la «Operación Matienzo», que consistió en el lanzamiento en simultáneo de cohetes Gamma Centauro y balones desde la base Matienzo en la Antártida y Chamical, a 3.950 km. de distancia, para la medición de la radiación cósmica, y el Proyecto $\mathrm{BIO}$, que logró lanzar en sus cohetes a seres vivos y recuperarlos sin que sufrieran ningún daño. En este último proyecto se destacaron las experiencias del ratón Belisario y el mono Juan.

Estas experiencias impulsaron investigaciones en varias universidades nacionales, donde se desarroIlaron experimentos espaciales que formaban parte de la carga útil de los cohetes de la Fuerza Aérea, brindando, a su vez, capacitación a ingenieros en el desarrollo de hardware espacial.

Luego de más de dos décadas de desarrollo tecnológico incremental ininterrumpido, que vinculaba a componentes ideológicos de modernización, industrialización y autonomía tecnológica, se logró un nivel alto de concentración de recursos humanos con conocimientos, experiencia y equipamiento que permitía dar un salto cuantitativo y cualitativo. A pesar de los vaivenes políticos, se logró consolidar cierta capacidad organizativa y relativa estabilidad institucional. Todo esto generó que "el desarrollo de cohetes se mantuviera en una curva más o menos ascendente hasta principios de la década de 1970, y a fines de esa década, argentina contaba con la posibilidad de desarrollar un vehículo lanzador de satélites de bajo costo" (De León, Hurtado, 2015).

Durante la última dictadura cívico-militar, sumado al conflicto limítrofe con Chile, la Fuerza Aérea modificó a línea de cohetería con objetivos científicos, hacia una de cohetes y misiles de carácter dual con claros intereses militares. Lo que posibilitó el desarrollo de misiles de la clase Alacrán y Cóndor.

\section{Regimen compensador (1982-1989)}

Luego de finalizada la Guerra de Malvinas, se abandonó la finalidad pacífica para redefinir como nueva prioridad el desarrollo de capacidades militares. Se impulsó la fabricación de un nuevo misil: el Cóndor II. Este misíl de alcance intermedio, concebido de forma ultrasecreta por la Fuerza Aérea, comenzó a desarrollarse en 1982.

En los últimos años de dictadura se produjeron una serie de cambios estratégicos y políticos del régimen tecnopolítico autonomista que llevaron a su reemplazo por un régimen de tipo compensador: el país comenzó a adquirir la tecnología necesaria en el extranjero y abandonar el desarrollo incremental local que llevaba casi dos décadas. Se interpretaba que había un atraso tecnológico con respecto a los países centrales y que era necesario la compra del know-how, los insumos básicos y las maquinarias necesarias para su producción para luego generar un desarrollo nacional.

De león (2015) vislumbra que durante esta etapa se produjo un fuerte conflicto entre los militares y civiles 
que habían completado sus estudios en el exterior, y aquellos que habían desarrollado la totalidad de su carrera en el país. Los primeros, tenían una visión de los recursos económicos de infraestructura y de las capacidades científico-tecnológicas de los Estados Unidos y Europa y veían las carencias del medio local, al punto de dudar de la capacidad de los centros científicos y tecnólogos nacionales para realizar proyectos de alta complejidad en el área espacial. Por su parte, los militares, ingenieros y técnicos de formación puramente nacional, se oponían a la compra de "cajas negras", donde no había un aprendizaje real, y pensaban que todo podía hacerse mejor, más rápido y con menores costos aprovechando los recursos y el intelecto local.

La endeble economía del país en los años 80, puso en peligro la continuación de todos los proyectos aeroespaciales y durante el gobierno de Alfonsín se produjo una reducción de fondos para las Fuerzas Armadas, como consecuencia se detuvieron todas las experiencias científicas atmosféricas con cohetes de fabricación nacional. El proyecto Condor II continuó, aunque en el marco de la hiperinflación fue direccionado a la generación de divisas a través de la exportación. Allí comenzaron las presiones internacionales para su desmantelamiento.

\section{Etapa dependentista (1989-2003)}

Con la llegada de Carlos Menem a la presidencia, y la adopción de políticas neoliberales se produjo un nuevo cambio de régimen en las políticas aeroespaciales al que podemos llamar como "dependentista".

A partir de la presión de Estados Unidos, se canceló y desmanteló el proyecto "Cóndor II". Incluso se entregaron todos los componentes del cohete y la tecnología para construir motores de combustibles sólidos que se encontraban en la planta de Falda del Carmen (Córdoba), por lo que perdimos la capacidad no sólo de fabricar cualquier tipo de misil sino además la capacidad de fabricar lanzadores de satélites. Además, esto ocasionó la dispersión del equipo humano de técnicos e ingenieros altamente capacitados.

Como consecuencia se disolvió la CNIE y se creó la Comisión Nacional de Actividades Espaciales, CONAE, la cual sale de la esfera militar y es dirigida por civiles, en su mayoría científicos. A partir de 1994, se consolida una nueva política espacial orientada a proyectos satelitales de carácter científico. La CONAE tuvo un rol más bien secundario en este período, de gestor y articulador entre los actores que se encargaron de los desarrollos necesarios.

Durante esta etapa los principales desarrollos se dieron como resultado de una fuerte articulación con la NASA como el programa SAC, que puso en órbita 3 satélites: SAC-A, B y C en el período 19962000.

Se instaló la Estación Terrena Córdoba en 1996 para la recepción de datos, seguimiento, telemetría y control de satélites y se montó un laboratorio de integración y ensayos en el Centro Espacial Teófilo Tabanera. Se desarrolló conceptualmente un satélite de radar de baja potencia (SAOCOM 1) y se organizó el Instituto de Altos Estudios Espaciales Mario Gulich. 
| Ciencia, Tecnología y Política | Año 2 | N² | Enero-Junio 2019 | ISSN 2618-3188 | www.revistas.unlp.edu.ar/CTyP |

\section{Periodo autonomista (2003-2015)}

Durante los gobiernos de Néstor y Cristina Kirchner, se dió un nuevo giro político, económico y social que le dió un notable impulso a la ciencia y la tecnología. Se aumentaron fuertemente los presupuestos universitarios y de todos los organismos vinculados al desarrollo científico-tecnológico, se aumentó el numero de becarios e ingresos a la carrera del Investigador Científico y Tecnológico del CONICET y se creo el Ministerio de Ciencia, Tecnología e Innovación Productiva

El sector aeroespacial no fue la excepción: CONAE fue la encargada de elaborar el Plan Nacional Espacial (2004-2015), cuya ejecución se consolidó como una "política de estado". La CONAE adquiere un rol preponderante en el sensado, generación, transmisión, procesamiento, almacenamiento, distribución y uso de la información espacial.

Se crea la empresa estatal ARSAT, que absorbe los activos de Nahuelsat S.A, sus posiciones orbitales, satélites y su estación terrena en Benavídez. A partir de allí la empresa diseña la plataforma ARSAT-3K y encarga a INVAP la fabricación de los satélites ARSAT-1 y ARSAT-2. Tal tarea involucró a cientos de pymes de base tecnológica así como también a grupos de investigación del sector científico. Se creó el Centro de Ensayos de Alta Tecnología S.A (CEATSA) para realizar las pruebas y se obtuvieron certificados de calidad a nivel internacional. Ambos satélites son lanzados en 2014 y 2015 respectivamente, contando con 15 años de vida útil cada uno. Un verdadero hito para nuestro país.

A diferencia de la década de los 90, en la cual se lanzaron varios satélites en vinculación con la NASA con objetivo de proveer información necesaria para el sistema científico mundial, esta etapa apostó a la producción de satélites geoestacionarios, desarrollados y testeados en el país, que cumplieran un rol estratégico en materia de telecomunicaciones. Esto generó capacidad instalada para la producción de nuevos satélites, formación y retención de recursos humanos con alta especialización, capacidad para operar y comercializar las bandas telecomunicacionales siguiendo intereses nacionales (como por ejemplo la creación de la Televisión Digital Abierta).

Paralelamente, el Instituto de Investigaciones Científicas y Técnicas para la Defensa (Citedef) impulsó el proyecto de los cohetes Gradicom I y II (primer cohete de dos etapas de desarrollo nacional) y CONAE impulsó el lanzador Tronador. Este último con la finalidad de tener la capacidad propia de inyectar satélites de hasta $250 \mathrm{~kg}$ en órbitas de hasta 600 kms de altura. Paradójicamente, ya contábamos con desarrollos en combustibles sólidos para la propulsión de cohetes, pero dicha tecnología se perdió luego del desmantelamiento del proyecto Cóndor. Para el Tronador se optó por desarrollar la tecnología de lanzadores con combustibles líquidos, la cual es más sencilla pero tiene menos prestaciones, mientras que los Gradicom contaron con un nuevo desarrollo en combustibles sólidos.

El camino recorrido del Tronador para lograr madurez tecnológica comprendió el lanzamiento exitoso de los cohetes-sonda Tonador 1 (550 kg de empuje) y Tronador 1B (1,5 tn de empuje) en los años 2007 y 2008 respectivamente. Le siguió la prueba del vehículo experimental VEx1B de 4 Tn de empuje en 2014 para testear los sistemas de propulsión, navegación, guiado y control, todos de fabricación nacional. 


\section{Un nuevo periodo dependentista (2015-2019)}

Al asumir Mauricio Macri la presidencia se produce un nuevo giro en el sector aeroespacial. En este período, el Poder Ejecutivo aplica un fuerte ajuste presupuestario que paraliza los proyectos en curso e impulsa nuevamente medidas dependentistas, como un intento de vinculación entre ARSAT y la empresa Hughes para la fabricación de ARSAT-3 (Krakowiak, 19/08/2016) y la contratación de la consultora McKinsey para definir el plan de negocios de ARSAT (Krakowiak, 11/07/16), entre otras.

Paralelamente, se avanzó hacia una política de cielos abiertos en la cual se autorizó a más de 14 satélites extranjeros a operar en el país, y a que compitan directamente con ARSAT 1 y 2 (Krakowiak, 15/08/16). Mediante un decreto, se privatizaron las bandas de frecuencia de 3G y 4G del espacio radioeléctrico (asignadas de forma exclusiva a ARSAT) para favorecer a las empresas de Telecomunicaciones.

Finalmente, ARSAT-3 se abandonó completamente y se alquiló un viejo satélite a la empresa SES con el único fin de no perder la posición orbital 81 Oeste (Krakowiak, 11/02/2019). Por otro lado el proyecto tronador también frenó el desarrollo de motores y VENG se encuentran en la búsqueda de un motor de origen ruso (Cavataio, 2018).

Quizás el único hecho positivo de relevancia en materia aeroespacial haya sido la culminación del satélite SAOCOM-1A por parte de la CONAE y su exitosa puesta en funcionamiento.

\section{Conclusiones}

El sector aeroespacial, por su carácter dual, se encuentra mas inmerso en las complejidades de la política internacional, que en los asépticos centros de ensayos. Esta razón explica los distintos cambios sufridos a lo largo de toda su historia. Pero, a la vez, esa misma dualidad, le permitió al sector sobrevivir tanto a gobiernos desarrollistas como militaristas.

El régimen dependentista está demostrando, una vez más, que nos aleja del camino de producción de conocimientos valiosos y nos convierte en meros consumidores de tecnología. Este modelo disminuye el grado de autonomía necesario para generar innovaciones e interrumpe la estrategia incremental de los regímenes autonomistas.

La experiencia compensadora fue breve y en un contexto de crisis económica, igualmente podemos afirmar que no logró avanzar en el objetivo propuesto; adquirir tecnología de punta para contrarrestar la brecha tecnológica y luego volver al desarrollo autónomo. Se produjo todo lo contrario, los técnicos no ampliaron su know-how, y se frenaron los desarrollos nacionales autónomos.

Por su parte, los regímenes autonomistas lograron una senda de desarrollo que en pocos años empezó a dar sus frutos. El punto crucial de estos regímenes es ajustar el grado de autonomía posible. Se podría buscar generar intercambios de tecnología basados en la cooperación entre países, aprovechando experiencias locales.

Por último, en Argentina es necesario que la discusión por el modelo político incorpore en su cen- 
tralidad la discusión de régimen tecnopolítico, para lograr el pasaje de políticas públicas aisladas a políticas de estado que se mantengan a lo largo del tiempo. Esta es la única manera de asegurar la continuidad necesaria de los mismos, más allá de las administraciones circunstanciales. Para lo cual, es necesario una mayor participación de los sectores involucrados en el plano productivo y científico, y en la concientización sobre la importancia de estas políticas para el futuro del desarrollo tecnológico y económico de nuestro país.

\section{Bibliografía}

ARSAT. (2014). El sector espacial argentino: Instituciones referentes, proveedores y desafíos. Disponible en: https://www.arsat.com.ar/post/el_sector_espacial_argentino

Bilmes, G. (2012). Sobre la posibilidad de construir un estilo tecnológico en común en Latinoamérica. Cátedra Libre "Ciencia, Política y Sociedad". UNLP. http://blogs.unlp.edu.ar/catedracps/

Cavataio, P. (2018) NPO Energomash y CONAE discuten la provisión de motores para el Tronador. Latam Satelital. (28 de Abril de 2018) Recuperado de http://latamsatelital.com/npo-energomash-conae-discuten-la-provision-motores-tronador/

De León, P. (2015). El proyecto misilístico Cóndor. Su origen, desarrollo y cancelación (Tesis Doctoral).Disponible en:http://repositorio.udesa.edu.ar/jspui/handle/10908/10945

Hecht, G. (1998). The Radiance of France. Nuclear Power and National Identity After World War II. Cambridge: The MIT Press.

Krakowiak, F. (2016a) Macri comienza a blanquear a McKinsey. Página/12.(11 de Julio de 2016) Recuperado dehttps://www.pagina12.com.ar/diario/economia/2-303911-2016-07-11.html

Krakowiak, F. (2016b) Presionan por cielos abiertos satelitales.Página/12. (15 de Agosto de 2016) Recuperado dehttps://www.pagina12.com.ar/diario/economia/2-306871-2016-08-15.html

Krakowiak, F. (2016c) El PRO busca desregular hasta el cielo. Página/12. (19 de Agosto de 2016) Recuperado dehttps://www.pagina12.com.ar/diario/economia/2-307278-2016-08-19.html

Krakowiak, F. (2019) El costo de haber abandonado ARSAT-3.Página/12.(2 de Febrero de 2019) Recuperado dehttps://www.pagina12.com.ar/174238-el-costo-de-haber-abandonado-el-arsat-3

Lukin, T. (2012) Lanzamiento exitoso del Gradicom. Página/12, (12de Julio de 2012) Recuperado de https:// www.pagina12.com.ar/diario/elpais/1-172070-2011-07-12.html

Vera, M. Guglielminotti, C. y Moreno, C.(2015). La participación de la Argentina en el campo espacial: panorama histórico y actual.Ciencia, Docencia y tecnología16 (51). Recuperado de http://www.pcient.uner.edu. ar/index.php/cdyt/article/view/89/0 